\title{
THE FLUID PROFILE DURING SPIN-COATING OVER A SMALL SINUSOIDAL TOPOGRAPHY
}

\author{
M. A. HAYES and S. B. G. O'BRIEN
}

Received 15 August 2003

\begin{abstract}
We consider spin-coating over a small sinusoidal topography modelling the physical problem of the coating of a television screen. This involves depositing a phosphor layer on a substrate with a precoating consisting of small parallel striations. Despite the fact that the basic flow is radial, we show that the final liquid coating does not have radial variation; rather, it varies according to the underlying topography. We use a thin film model resulting in an evolution equation for the fluid thickness and sketch several techniques for obtaining approximate solutions in appropriate limiting situations.
\end{abstract}

2000 Mathematics Subject Classification: 76D45, 76D08, 76A20, 34E10.

1. Introduction. Spin-coating involves placing a suspension on a substrate which is then rotated. As a result of centrifugal effects, the liquid flows radially away from the centre $[1,2,3,6,10,11,12,14,15,18,19,20,23,27,28]$. The suspension consists of the colloid material to be deposited on the substrate suspended in a solvent, for example, phosphor in water. The centrifugal forces are offset by viscous and surface tension effects. At the beginning of the process, the liquid height drops rapidly due to centrifugal action; thereafter, the liquid height decreases slowly as the dominant contributor to the liquid height reduction is evaporation. When all the solvent is removed by a combination of spinning and evaporation, all that remains is a coating profile of the colloid material. In the present paper, we develop a model to predict the fluid thickness during spin-coating over a substrate with a preexisting topography.

This process is of considerable importance in industry. When coating the inside of colour TV screens $[10,11,13]$ (the latter is a useful reference on spin-coating-the process used to coat the inside of colour TV screens), three different colour phosphors have to be applied. The finished product has a strip of the first colour phosphor approximately $1 \mathrm{~mm}$ wide running from the top to the bottom of the screen (see Figure 1.1). A strip of the second and third phosphor colours of similar width are applied adjacent to the first strip. This process is repeated across the entire screen. The first phosphor colour is applied using spin-coating on a flat topography. The space for the second and third phosphor colours is obtained by etching the profile of the first phosphor. The second and third phosphor colours are then applied using spin-coating over what is essentially a periodic topography. At the end of this paper, we use Fourier series techniques and the fluid thickness over a sinusoidal topography to find the fluid profile over an arbitrary even periodic topography. 


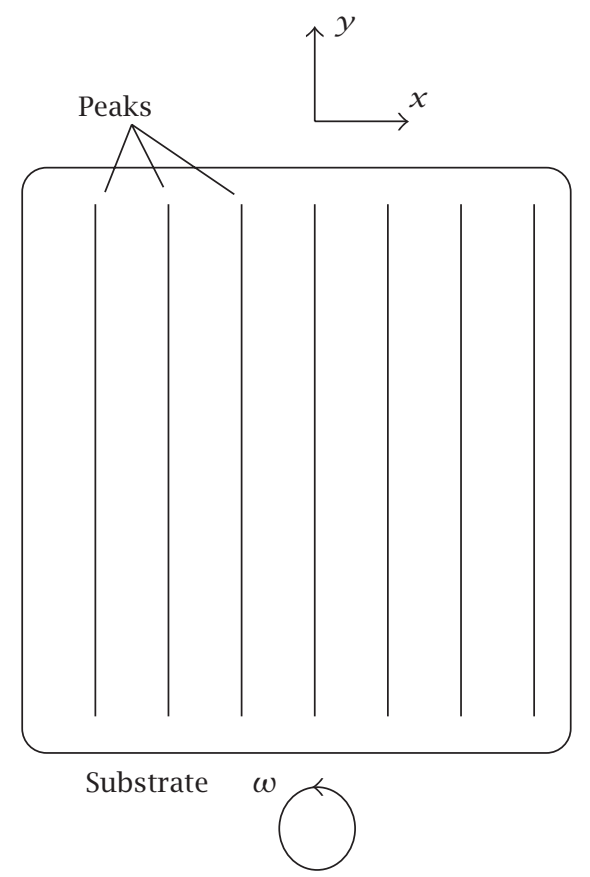

FIGURE 1.1. Schematic of the coating process. The substrate to be coated has a preexisting topography, the peaks of which are shown as parallel lines. The coating solution is placed on the substrate which is then rotated in order to spread the liquid.

Lammers $[10,11,12]$ numerically obtained the fluid and solute profiles during spincoating over repeated steps up and down including evaporation and both constant and variable surface tension (induced by Marangoni effects). Recent work by Homsy et al. $[4,8,29]$ has studied the stability of flows of this type and it has even suggested how capillary ridges in the free surface can be flattened using Marangoni effects.

In this paper, we apply lubrication theory [21, 26] and use a perturbation technique to solve for the fluid thickness over a small sinusoidal topography during spin-coating using a number of ad hoc analytical methods of solution. Firstly, we obtain solutions over different spatial domains assuming that the first-order perturbation of the fluid thickness is time independent. This is justified towards the end of the process when we would expect the rate of fluid reduction to be negligible in comparison to the rate of change in the flow direction. Secondly, we incorporate time dependence. We note that the basic spin effects induce a radial flow so, at first glance, we might expect a radially symmetric fluid profile. We will show in fact that the fluid profile is dependent only on the $x^{*}$ direction as outlined in Figure 1.1.

In Section 2, we develop the mathematical model. In Section 3, we obtain solutions using a variety of approximations. In Section 4, we show how the results may be generalised to the case of arbitrary topography. Finally, we make some closing observations. 
2. Mathematical model. The mathematical modelling of the physical process started with the work of Emslie et al. [3]. This analysis considers only Newtonian fluids, and takes advantage of the thinness of the film compared with the radial expanse of the substrate, justifying the use of a lubrication analysis. Emslie et al. neglected all forces except centrifugal and viscous; thus gravitational, coriolis, surface tension, and capillary forces are ignored as is air drag on the free surface. Neglect of these forces is usually justified except for uneven substrates, where capillary forces must be introduced. Less justifiable is the assumption of the constancy of fluid properties, most notably the unrealistic assumption of neglecting solvent evaporation. The effect of evaporation of the coating liquid was first addressed by Meyerhofer [19]. He included evaporation of the solvent in a model predicting quite successfully the final layer thickness of solute. Despite Meyerhofer's own opinion, his set of equations is amenable to analytical treatments. The main precursor to the work in the present paper was contributed by Lammers [10, 11, 12]. He modelled the slightly varying evaporation rate and solved Meyerhofer's model for nonuniform evaporation analytically (using perturbation theory) and numerically $[10,11,12]$. Lammers also solved the governing spin-coating equations numerically for the fluid and solute thickness over a nonflat substrate incorporating evaporation and constant and varying surface tension, including Marangoni effects.

The related problem of calculating the film thickness of thin film flow over topography caused by different external forces has been addressed by many authors. Extensive research contributions have been made on thin film flow in particular by Stillwagon and Larson [27] who worked on levelling of thin films over uneven substrates during spincoating. Other contributions on the spin-coating process include $[6,10,11,12,13,18$, $23,28]$ and gravity-driven flow $[5,7,22,24,25]$. In particular, the closely related work [7] considers the motion of a thin viscous film flowing over a trench or a mold. Lubrication theory is used to simplify the equations of motion to a nonlinear partial differential equation for the evolution of the free surface in time and space. Quasisteady solutions for the free surface are reported for different sized topographies, in particular, different depth, width, and steepness. The authors reveal that the free surface develops a ridge before the entrance to a trench (or exit from a mold) and this ridge can become large for steep substrate features of significant depth. Other related works [5, 25] compute the fluid thickness over an arbitrary topography using numerical and analytical methods, respectively. The results show, for an isolated mold type topography, that the fluid thickness reduces just before reaching the topography and a horseshoe-shaped risen wake appears in front of the topography. Other works [16, 17] consider the nonlinear evolution of small- and large-amplitude initial periodic disturbances on vortex tails.

Lawrence $[14,15]$ showed that the final coating thickness in spin-coating depends on the initial concentration of solute, the kinematic viscosity, the diffusion coefficient of the solute, and the spin speed, but is independent of the evaporation rate.

We define the typical fluid thickness to be $H$, the characteristic length scale (period of the topography) to be $L \gg H$; and $\epsilon$ is a small parameter defined by $\epsilon=H / L$. The substrate topography under consideration is assumed to take the form 


$$
T^{*}\left(x^{*}\right)=\epsilon H T\left(\frac{x^{*}}{L}\right) \text {. }
$$

We use Cartesian coordinates such that the $y^{*}$-independent topography shows sinusoidal variation in the $x^{*}$ direction, where the reference frame is rotating with the substrate (see Figure 1.1). The Navier Stokes equations for incompressible flow are

$$
\begin{gathered}
\left(\mathbf{u}^{*} \cdot \nabla\right) \mathbf{u}^{*}=-\frac{1}{\rho} \nabla p^{*}+v \nabla^{2} \mathbf{u}^{*}+\left(\omega^{2} x^{*}, \omega^{2} y^{*}, 0\right), \\
\operatorname{div}^{*}=0,
\end{gathered}
$$

where the velocity vector $\mathbf{u}^{*}=\left(u^{*}, v^{*}, w^{*}\right)$, the acceleration vector due to centrifugal induced spin effects, is included explicitly, $p^{*}$ is the the pressure, $\omega$ is the angular velocity, $\rho$ is density, and $v$ is the kinematic viscosity. We wish to simplify the Navier Stokes equations for the case of thin film flow. We nondimensionalise all variables according to the following scales (variables with asterisks are dimensional, uppercase symbols are dimensional and are of the order of their respective dimensional variables, and lowercase variables are nondimensional and are of order unity):

$$
\begin{gathered}
x^{*}=L x, \quad y^{*}=L y, \quad t^{*}=\frac{3}{2} \Lambda t, \quad p^{*}=P p, \quad u^{*}=U u, \\
v^{*}=U v, \quad w^{*}=W w, \quad z^{*}=H z, \quad h^{*}=H h .
\end{gathered}
$$

$z^{*}$ denotes the Cartesian coordinate in the direction perpendicular to the plane of fluid flow. $u^{*}, v^{*}$, and $w^{*}$ are the velocities in the $x^{*}, y^{*}, z^{*}$ directions, respectively. $h^{*}$ is the perpendicular distance from the $z^{*}=0$ plane to the top of the fluid. $\Lambda$ is the time scale for the process which will be defined precisely later. $U$ and $W$ are the typical in-plane and vertical velocities, respectively, with $W=\epsilon U$. We define $e_{v}$ to be the evaporation rate which is assumed to be independent of the film thickness [19] and we define a Reynolds number as $\mathfrak{R}=\left(H^{2} / L^{2}\right)(U L / v)=\epsilon^{2}(U L / v) \ll 1$ and neglect terms of $O\left(\mathfrak{R}, \epsilon^{2}\right)$ (though we retain terms of $O(\epsilon)$ which include the effects of the topography). We choose, as a pressure scale, $P=\mu U L / H^{2}$ and find that (2.2) can be approximated by

$$
0=-\frac{\partial p}{\partial x}+\frac{\partial^{2} u}{\partial z^{2}}+x, \quad 0=-\frac{\partial p}{\partial y}+\frac{\partial^{2} v}{\partial z^{2}}+y, \quad 0=-\frac{\partial p}{\partial z}
$$

if we define $U=H^{2} \omega^{2} L / v$.

2.1. The boundary conditions. We assume that the air exerts zero stress on the liquid surface, that is, on the free surface

$$
\mathbf{t}^{T} \mathbf{T n}=0
$$

where $\mathbf{n}$ is the unit normal vector at the fluid surface, $\mathbf{T}$ is the liquid stress tensor, and $\mathbf{t}$ is a tangential unit vector at the fluid surface. At the free surface given by $z^{*}=$ $h^{*}\left(x^{*}, y^{*}\right)$, it follows that $\mathbf{n}=\left(1 / \sqrt{1+h_{x}^{* 2}+h_{y}^{* 2}}\right)\left(-h_{x}^{*},-h_{y}^{*}, 1\right)$. In dimensionless form, we find, to $O\left(\epsilon^{2}\right)$, that

$$
\frac{\partial u}{\partial z}=0 ; \quad \frac{\partial v}{\partial z}=0 \quad \text { on } z=h(x, y)
$$


The no-slip condition in dimensionless form is

$$
u=v=0 \quad \text { on } z=\epsilon T \text {. }
$$

2.2. The evolution equation. From (2.5), (2.7), and (2.8), we find $\mathbf{u}$, the in-plane velocity, to be

$$
\mathbf{u}=\nabla p_{\text {dyn }}\left[\frac{z^{2}-(\epsilon T)^{2}}{2}-h(z-\epsilon T)\right]
$$

where $p_{\text {dyn }}$ denotes the dimensionless dynamic pressure including the centrifugal effects. We define the components of $\overline{\mathbf{u}}$ to be the in-plane velocities averaged over the film thickness so that

$$
\overline{\mathbf{u}}=-\nabla p_{\mathrm{dyn}} \frac{(h-\epsilon T)^{2}}{3}
$$

Applying a force balance normal to the fluid surface, we obtain

$$
\mathbf{n}^{T} \mathbf{T n}=\gamma \kappa,
$$

where $\gamma$ is the surface tension of the fluid and $\kappa$ is the curvature of the liquid free surface. In dimensionless form and incorporating the thin film approximation, this reduces to

$$
p=-B \nabla^{2} h
$$

where $B=\gamma H^{3} / \mu U L^{3}$ is assumed to be at least $O(\epsilon)$ in order to retain surface tension effects. Assuming incompressibility and including the evaporation rate $e_{v}$ scaled with the ratio of the typical fluid thickness to the process time $H / \Lambda$, we obtain the following evolution equation for the liquid free surface $h(x, y, t)$ :

$$
\frac{\partial h}{\partial t}+\frac{\partial(\bar{u}(h-\epsilon T))}{\partial x}+\frac{\partial(\bar{v}(h-\epsilon T))}{\partial y}+\frac{e_{v} \Lambda}{H}=0 .
$$

We assume that the Peclet numbers in the $x^{*}$ and $y^{*}$ directions are large so that convection dominates diffusion. Exploiting the thinness of the liquid film, we assume that the concentration of solute across the film is approximately constant, that is, the relevant Peclet number in the $z^{*}$ direction is assumed small. The diffusivity coefficient and the viscosity are also assumed constant though these may in fact be dependent on the solute concentration. However, in this approximation, the variation in the diffusion coefficients can be neglected because of the assumed Peclet numbers. In addition, it is now known [12] that in most practical coating applications, the flow has practically stopped as a result of convective thinning before the viscosity increases significantly. This indicates that the approximation originally made by Meyerhofer [19] and followed by many other authors, for example, as in [23], was essentially correct. Of course, improved models could take into account the small viscosity changes that can occur (this is discussed in [12]) but our aim here is to develop a leading-order model in the spirit of Meyerhofer and Lammers et al. 
If we define a flux via $\mathbf{q}=\left(x+B\left(h_{x x}+h_{y y}\right)_{x}, y+B\left(h_{x x}+h_{y y}\right)_{y}\right)(h-\epsilon T)^{3}$, the evolution equation can be written as

$$
\frac{\partial h}{\partial t}+\frac{1}{3} \nabla \cdot \mathbf{q}+\frac{e_{v} \Lambda}{H}=0
$$

Equation (2.14) contains one unknown dependent variable $h$, and will be used later to obtain the leading- and first-order perturbation equations of the scaled fluid thickness $h$. We linearise by substituting (2.13) into

$$
h(x, y, t)=h_{0}(t)+\epsilon h_{1}(x, y, t)
$$

and ignoring terms of $O\left(\epsilon^{2}\right)$ or smaller. This substitution is motivated by the fact that at leading order, that is, for spin-coating on a flat substrate, it is well known that regardless of the initial film thickness, the liquid quickly levels into a uniform film [3] whose thickness depends only on the time. In fact, the film thickness, after a short time, is more or less independent of the initial film thickness. In this model, we are thus ignoring the very early (and unimportant) stages of the process, during which the film quickly equilibrates. The directional variation in our model arises at the next order via the effects of the topography. Hence we find that

$$
\begin{aligned}
\frac{d h_{0}}{d t}+\epsilon \frac{\partial h_{1}}{\partial t}=-h_{0}^{3}-\frac{3 e_{v} \Lambda}{2 H}+\epsilon[ & -\frac{3}{2} x h_{0}^{2} \frac{\partial\left(h_{1}-T\right)}{\partial x}-\frac{3}{2} y h_{0}^{2} \frac{\partial\left(h_{1}-T\right)}{\partial y} \\
& \left.-3 h_{0}^{2}\left(h_{1}-T\right)-\frac{B}{2} h_{0}^{3} \nabla^{2}\left(\nabla^{2} h_{1}\right)\right] .
\end{aligned}
$$

Following previous authors, for example, Meyerhofer [19], we assume that the evaporation rate is independent of the concentration. In practical situations, the coating process is begun with an excess of dilute solution (in which case, the final coating thickness is independent of the initial film height). The process separates approximately into two stages. During the first stage, the film thins primarily because of spin effects and evaporative effects are virtually negligible. During this stage, the evaporation rate can be taken to be approximately constant, as the solute concentration remains at its initial value. As the film becomes thinner, the flow slows down until finally (from a practical point of view) further thinning only progresses via evaporation with subsequent increase in concentration (spin effects are practically zero at this point). If the evaporation rate is strongly concentration-dependent during this phase, the assumption of constant evaporation rate is, strictly speaking, incorrect, but in fact, this will not lead to any error in predicting the final layer thickness, the primary aim of this analysis (though it will incorrectly predict the overall process time). This is the reason that the constant evaporation model of Meyerhofer [19] gives results for the final film thickness in agreement with experiment; we will adopt the same approach. Of course, if the evaporation rate $e_{v}$ is known as a function of the concentration, this can be easily incorporated into the model to obtain improved estimates for the overall process time. 
We now choose $H=h_{0}^{*}$ and $\Lambda$ such that $3 e_{v} \Lambda / 2 H=1$ so that (2.16) yields

$$
\begin{gathered}
\frac{d h_{0}}{d t}=-h_{0}^{3}-1, \\
\frac{\partial h_{1}}{\partial t}=-\frac{3}{2} x h_{0}^{2} \frac{\partial\left(h_{1}-T\right)}{\partial x}-\frac{3}{2} y h_{0}^{2} \frac{\partial\left(h_{1}-T\right)}{\partial y}-3 h_{0}^{2}\left(h_{1}-T\right)-\beta h_{0}^{3} \nabla^{2}\left(\nabla^{2} h_{1}\right),
\end{gathered}
$$

where $\beta=\gamma H / 2 \rho \omega^{2} L^{4}$ and $1<\beta<1 / \epsilon$ since, typically, $\gamma=4 \times 10^{-2} \mathrm{Kg} / \mathrm{s}^{2}, H=10^{-5} \mathrm{~m}$, $\rho=10^{3} \mathrm{Kg} / \mathrm{m}^{3}, \omega=10 \mathrm{~s}^{-1}$, and $L=10^{-3} \mathrm{~m}$.

It is an elementary exercise to show that we also obtain (2.18) if we start with the spin-coating equation in its more familiar cylindrical coordinate form:

$$
\frac{\partial h^{*}}{\partial t^{*}}=-\frac{1}{3 r^{*}} \frac{\rho \omega^{2}}{\mu} \frac{\partial}{\partial r^{*}}\left(r^{* 2}\left(h^{*}-T^{*}\right)^{3}\right)-\frac{\gamma}{3 \mu} \nabla \cdot\left(\left(h^{*}-T^{*}\right)^{3} \nabla\left(\nabla^{2} h^{*}\right)\right)-e_{v},
$$

and then make the assumption that $h=h_{0}(t)+\epsilon h_{1}(x, y, t)$. We note that if $L$ (the period of the topography) is very large, the topography is effectively flat and the problem simplifies to solving (2.17), that is, a solution uniform in $x$ and $y$ and dependent on $t$ only.

Solving (2.17) using an infinite initial condition, we obtain the following base-state solution for $h_{0}$ :

$$
t=-\frac{1}{3} \ln \left(\frac{h_{0}+1}{\sqrt{h_{0}^{2}-h_{0}+1}}\right)-\frac{1}{\sqrt{3}} \arctan \left(\frac{2 h_{0}-1}{\sqrt{3}}\right)+\frac{\pi}{2 \sqrt{3}},
$$

where we use the principal value of the arctan function.

2.2.1. A solution strategy. In Figure 1.1, we consider a rotating substrate. We are interested in the case where we have a low-amplitude, long-period sinusoidal topography uniform with respect to the $y^{*}$ direction (see Figure 1.1). We thus assume that $T^{*}\left(x^{*}\right)=\epsilon H \cos \left(2 \pi x^{*} / L\right)$ so that $T(x)=\cos (2 \pi x)$. In Figure 1.1, the peaks of the topography are shown as straight lines.

The central line is defined as the line passing through the centre of rotation in the plane of the substrate parallel to the peaks of the topography, that is, the line $x=0$ (see Figure 1.1). As the centrifugal force acting on the fluid is proportional to the distance from the centre of rotation, the $x$ component of this force is proportional to $x$ and is constant along a line of constant $x$. As the topography is dependent on $x$ only, the topographical disturbance to the flow will be constant on a line of constant $x$. According to the lubrication approximation, the velocity responds linearly to the driving force which results in the $x$ component of velocity being constant on a line of constant $x$ (i.e., on a line parallel to the central line).

For a line of constant $x$, the $y$ component of centrifugal force and consequently the velocity in the $y$ direction are linear in $y$. Hence, from (2.10), we see that the fluid thickness must be constant on a line of constant $x$ (equivalently, the pressure will not vary in the $y$ direction as can be seen from (2.12)). 
Hence, we look for translation-invariant solutions for the fluid profile which depend on $x, t$ only. Though the topography is independent of $y$, the basic spin effects induce a radial flow; so at first sight, we might not expect such a $y$-independent film thickness. However, because the base flow is position independent, it is clear that we can simplify by assuming that $h_{1}=h_{1}(x, t)$, and (2.18) now yields

$$
\frac{\partial h_{1}}{\partial t}=-\frac{3}{2} x h_{0}^{2} \frac{\partial\left(h_{1}-T\right)}{\partial x}-3 h_{0}^{2}\left(h_{1}-T\right)-\beta h_{0}^{3} \frac{\partial^{4} h_{1}}{\partial x^{4}}
$$

We will use the initial condition $h_{1}(x, 0)=0$. The correctness of the form of $h_{1}(x, t)$ is verified by the fact that (2.21) is $y$ independent and no contradiction arises.

3. Solutions. Since (2.21) has nonconstant coefficients, we cannot find a solution using standard techniques such as separation of variables, and a full solution can only be found numerically. Instead we set ourselves the task of constructing an approximate analytic solution in an ad hoc fashion. For small perpendicular distances from the centre line, the $x$ component of centrifugal force is small and the surface tension effect is dominant, so the amplitude of the fluid profile is small in this region. At larger distances, the $x$ component of centrifugal force is large in comparison to the surface tension effect and, as a result, the fluid profile will be almost conformal to the topography. For these reasons and from what is known from experimental works [10, 11, 12], we predict that the disturbance in the fluid thickness for small $x$ will consist of small sinusoidal oscillations whose amplitude increases approximately linearly with $x$, while for large $x$, it will become almost conformal.

Consequently, we will use the following ansatz to solve (2.21):

$$
h_{1}=A(x, t) x \sin (2 \pi x)+B(x, t) \cos (2 \pi x) .
$$

We first solve for $h_{1}$ and assume $A(x, t)$ and $B(x, t)$ are independent of time and have negligible space derivatives. Subsequently, we assume $A(x, t)$ and $B(x, t)$ are independent of time and the solution is only valid for $x \ll \beta$, though spatial derivatives are included.

Then we incorporate the time dependence and the spatial derivatives of $A(x, t)$ and $B(x, t)$ in the solution procedure for $x \ll \beta$. Finally, the time dependence of $A(x, t)$ and $B(x, t)$ is included in the solution procedure and the solutions are valid for all $x$. In this case, the spatial derivatives are assumed negligible; we will justify this a posteriori.

3.1. Quasistatic solutions. We solve for the first-order perturbation term of the fluid thickness assuming it is independent of time. This assumption is reasonable towards the end of the process when $h_{1}$ and consequently $A(x, t)$ and $B(x, t)$ are changing least rapidly. Physically, this corresponds to the situation where the liquid level is thinning slowly relative to the speed with which the profile adjusts to an underlying topography.

In this section, we obtain solutions for $h_{1}$ over different spatial domains and for $t \gg 1$ when the flow is quasisteady. 
3.1.1. Solutions for $t \gg 1$. The space derivatives of $A(x)$ and $B(x)$ are assumed to be negligible which we will verify a posteriori. On substituting the topography $T(x)=$ $\cos (2 \pi x)$ and (3.1) into the governing equation for $h_{1}$ (2.21), we obtain

$$
\begin{aligned}
0= & \left(-\frac{9}{2} h_{0}^{2} A+3 \pi h_{0}^{2} B-16 \pi^{4} \beta h_{0}^{3} A-3 h_{0}^{2} \pi\right) x \sin (2 \pi x) \\
& +\left(-3 h_{0}^{2} B-3 \pi x^{2} h_{0}^{2} A-16 \pi^{4} \beta h_{0}^{3} B+32 \pi^{3} \beta h_{0}^{3} A+3 h_{0}^{2}\right) \cos (2 \pi x) .
\end{aligned}
$$

Equating the coefficients of sine and cosine on the right-hand side of (3.2) to zero, we obtain two linear equations in $A(x)$ and $B(x)$ whose solutions are

$$
\begin{aligned}
& A(x)=\frac{-(16 / 3) \pi^{3} h_{0} \beta}{3 / 2 \pi^{2}+(8 / 3) \pi^{2} h_{0} \beta+(256 / 9) \pi^{6} h_{0}^{2} \beta^{2}+x^{2}}, \\
& B(x)=\frac{3 / 2 \pi^{2}-(16 / 3) \pi^{2} h_{0} \beta+x^{2}}{3 / 2 \pi^{2}+(8 / 3) \pi^{2} h_{0} \beta+(256 / 9) \pi^{6} h_{0}^{2} \beta^{2}+x^{2}} .
\end{aligned}
$$

In (3.3), $h_{0}$ is assumed independent of $t$ for $t \gg 1$; the flow is assumed to have effectively stopped at this point. From (3.3), it can be seen that $A(x)$ takes a finite negative value when $x=0$ and increases asymptotically to zero as $x \rightarrow \infty$, while $B(x)$ assumes a value close to zero when $x=0$ and increases asymptotically to unity as $x \rightarrow \infty$. This behaviour of $A(x)$ and $B(x)$ is expected from physical considerations [10,11, 12]. We assumed that spatial derivatives of $A(x)$ and $B(x)$ up to and including the fourth order were negligible. For $h_{0}=1$ and $\beta=5$, the maximum absolute value of the first four spatial derivatives of $A(x)$ and $B(x)$ is $<10^{-4}$.

3.1.2. Solution for $x \ll \beta, t \gg 1$. We solve for $h_{1}(x, t)$ while now including the effect of the spatial derivatives. To evaluate these, $A(x)$ and $B(x)$ are written in a Taylor series in powers of $x / \beta$. From the previous subsection, $A(x)$ can be written as

$$
A(x)=\frac{a_{1} \beta}{b_{1}+c_{1} \beta+d_{1} \beta^{2}+x^{2}} .
$$

Equation (3.4) can be expressed as a polynomial when $x \ll \beta$ :

$$
A(x)=\frac{1}{\beta}\left(A_{1}+A_{2} \frac{1}{\beta}+A_{3} \frac{1}{\beta^{2}}+A_{4} \frac{x^{2}}{\beta^{2}}\right),
$$

truncating so that terms of $O\left(1 / \beta^{2}\right)$ or larger are included. Similarly, $B(x)$ can be written as

$$
B(x)=\frac{a_{2}+b_{2} \beta+x^{2}}{c_{2}+d_{2} \beta+e_{2} \beta^{2}+x^{2}},
$$

which can be expressed as

$$
B(x)=\frac{1}{\beta}\left(B_{1}+B_{2} \frac{1}{\beta}+B_{3} \frac{1}{\beta^{2}}+B_{4} \frac{x^{2}}{\beta^{2}}+B_{5} \frac{x^{2}}{\beta}\right) .
$$

The spatial derivatives of $A(x)$ and $B(x)$ can be easily obtained from (3.5) and (3.7). Substituting (3.1) into (2.21), we obtain 14 groups of terms. Each group will consist of 
terms which can be a function of $h_{0}, A_{i}, B_{j}, i=1, \ldots, 4, j=1, \ldots, 5$, with factors involving powers of $\beta$ and $x$. As the left-hand side of (2.21) equals zero and the common factors in each group are independent, all of these groups must equal zero. We thus obtain 9 linearly independent equations in the 9 unknowns $A_{i}, B_{j}$ :

$$
\begin{aligned}
& 0=-3 \pi-16 \pi^{4} h_{0} A_{1}, \\
& 0=3+32 \pi^{3} h_{0} A_{1}-16 \pi^{4} h_{0} B_{1}, \\
& 0=-3 \pi A_{1}-16 \pi^{4} h_{0} B_{5}, \\
& 0=3 \pi B_{5}-16 \pi^{4} h_{0} A_{4}, \\
& 0=-\frac{9}{2} A_{1}+3 \pi B_{1}-64 \pi^{3} h_{0} B_{5}-16 \pi^{4} h_{0} A_{2}, \\
& 0=-6 B_{5}-3 \pi A_{2}+96 \pi^{3} h_{0} A_{4}-16 \pi^{4} h_{0} B_{4}, \\
& 0=-3 B_{1}+48 \pi^{2} h_{0} B_{5}+32 \pi^{3} h_{0} A_{2}-16 \pi^{4} h_{0} B_{2}, \\
& 0=-\frac{9}{2} A_{2}+3 \pi B_{2}+144 \pi^{2} h_{0} A_{4}-64 \pi^{3} h_{0} B_{4}-16 \pi^{4} h_{0} A_{3}, \\
& 0=-3 B_{2}-48 \pi h_{0} A_{4}+48 \pi^{2} h_{0} B_{4}+32 \pi^{3} h_{0} A_{3}-16 \pi^{4} h_{0} B_{3} .
\end{aligned}
$$

Equations (3.8) can be solved to express $A_{i}$ and $B_{j}$ in terms of $h_{0}$. Thus from (3.4), (3.5), (3.6), and (3.7), we find that

$$
\begin{aligned}
& A(x)=\frac{-(16 / 3) \pi^{3} h_{0} \beta}{-13 / 2 \pi^{2}-(56 / 3) \pi^{2} h_{0} \beta+(256 / 9) \pi^{6} h_{0}^{2} \beta^{2}+x^{2}}, \\
& B(x)=\frac{7 / 2 \pi^{2}-(16 / 3) \pi^{2} h_{0} \beta+x^{2}}{9 / 2 \pi^{2}-(104 / 3) \pi^{2} h_{0} \beta+(256 / 9) \pi^{6} h_{0}^{2} \beta^{2}+x^{2}} .
\end{aligned}
$$

Equations (3.9) are very similar to the solution for $A(x)$ and $B(x)$ in Section 3.1.1. This similarity remains the case when $x>\beta$ despite the restriction on $x$ in the current method of solution.

To show the similarity between solutions resulting from the two methods, we plot $h_{1}$ for the two time-independent solution methods together with two time-dependent solutions in Figures 3.1, 3.2, 3.3, and 3.4.

\subsection{Time-dependent solutions}

3.2.1. Solution for $x \ll \beta$ and all $t$. In the previous section, we neglected time dependence. In this subsection, we solve in the region $x \ll \beta$ for the first-order perturbation term of the fluid thickness including time dependence and the effect of the spatial derivatives. As in the previous subsection, we express $A(x, t)$ and $B(x, t)$ as polynomials in $x$ for $x \ll \beta$. Rewriting (3.5) and (3.7), incorporating the time dependence in $A_{i}$, $B_{j}$, we have

$$
\begin{aligned}
& A(x, t)=\frac{1}{\beta}\left(A_{1}(t)+A_{2}(t) \frac{1}{\beta}+A_{3}(t) \frac{1}{\beta^{2}}+A_{4}(t) \frac{x^{2}}{\beta^{2}}\right), \\
& B(x, t)=\frac{1}{\beta}\left(B_{1}(t)+B_{2}(t) \frac{1}{\beta}+B_{3}(t) \frac{1}{\beta^{2}}+B_{4}(t) \frac{x^{2}}{\beta^{2}}+B_{5}(t) \frac{x^{2}}{\beta}\right) .
\end{aligned}
$$


THE FLUID PROFILE DURING SPIN-COATING ...

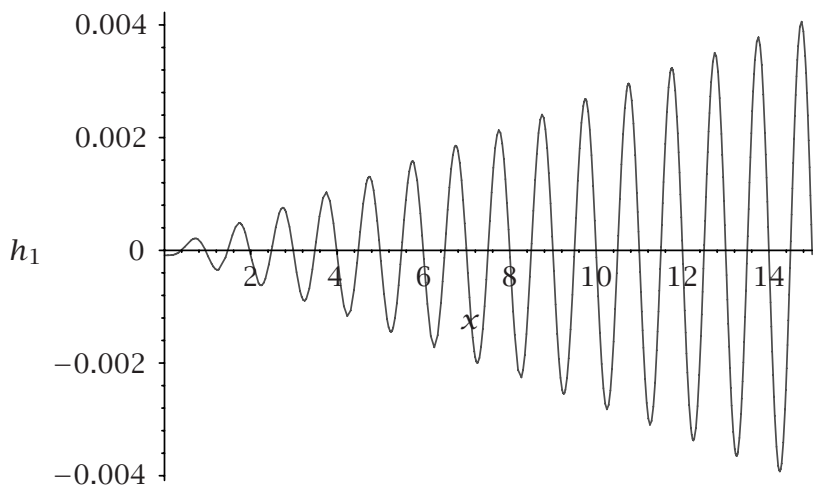

FIGURE 3.1. $h_{1}$ for $t=0.1, \beta=10$.

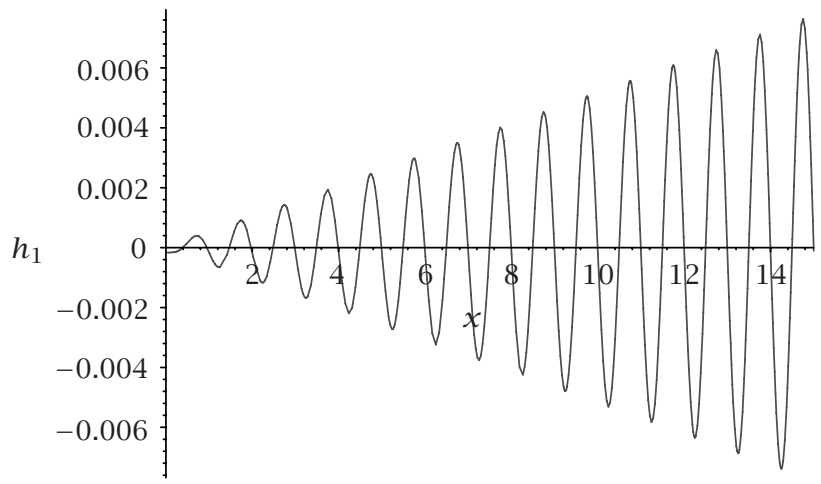

FiguRE 3.2. $h_{1}$ for $t=0.3, \beta=10$.

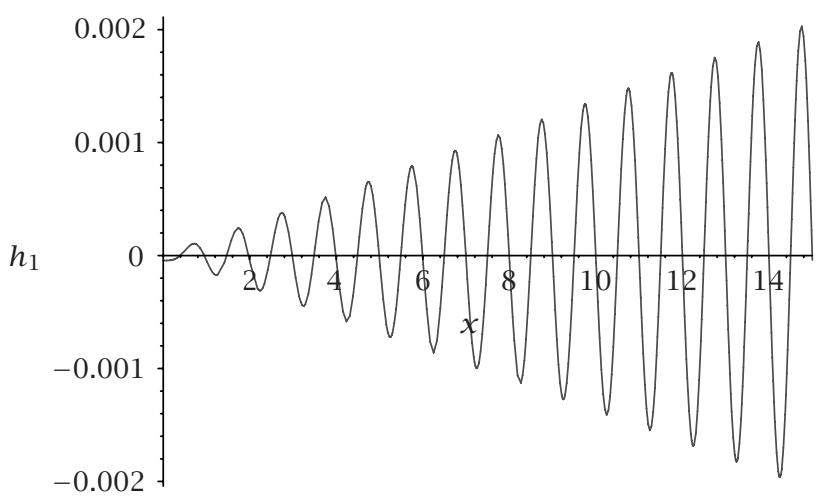

FiguRE 3.3. $h_{1}$ for $t=0.1, \beta=20$. 


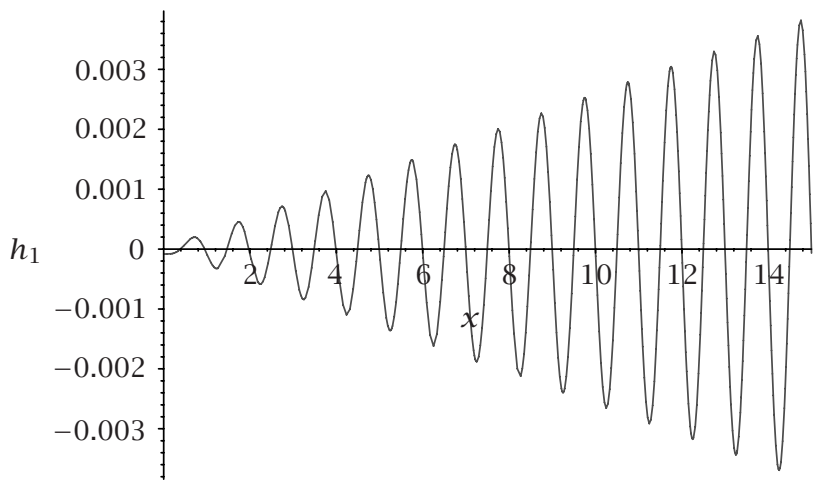

FIGURE 3.4. $h_{1}$ for $t=0.3, \beta=20$.

Substituting (3.10) into (3.1) and using (2.21), we obtain

$$
\begin{aligned}
\frac{1}{\beta}\left(\frac{d A_{1}}{d t}+\frac{d A_{2}}{d t} \frac{1}{\beta}+\frac{d A_{3}}{d t} \frac{1}{\beta^{2}}+\frac{d A_{4}}{d t} \frac{x^{2}}{\beta^{2}}\right) x \sin (2 \pi x) \\
+\frac{1}{\beta}\left(\frac{d B_{1}}{d t}+\frac{d B_{2}}{d t} \frac{1}{\beta}+\frac{d B_{3}}{d t} \frac{1}{\beta^{2}}+\frac{d B_{4}}{d t} \frac{x^{2}}{\beta^{2}}+\frac{d B_{5}}{d t} \frac{x^{2}}{\beta}\right) \cos (2 \pi x) .
\end{aligned}
$$

Again from (3.1) and (2.21), we obtain 14 groups of terms identical to those in the previous section. On comparing to (3.11), we obtain 9 linearly independent first-order ordinary differential equations in $A_{i}, B_{j}$ as follows:

$$
\begin{aligned}
0 & =-3 \pi-16 \pi^{4} h_{0} A_{1}, \\
0 & =3+32 \pi^{3} h_{0} A_{1}-16 \pi^{4} h_{0} B_{1}, \\
0 & =-3 \pi A_{1}-16 \pi^{4} h_{0} B_{5}, \\
0 & =3 \pi B_{5}-16 \pi^{4} h_{0} A_{4}, \\
\frac{d A_{1}}{d t} & =-\frac{9}{2} A_{1}+3 \pi B_{1}-64 \pi^{3} h_{0} B_{5}-16 \pi^{4} h_{0} A_{2}, \\
\frac{d B_{5}}{d t} & =-6 B_{5}-3 \pi A_{2}+96 \pi^{3} h_{0} A_{4}-16 \pi^{4} h_{0} B_{4}, \\
\frac{d B_{1}}{d t} & =-3 B_{1}+48 \pi^{2} h_{0} B_{5}+32 \pi^{3} h_{0} A_{2}-16 \pi^{4} h_{0} B_{2}, \\
\frac{d A_{2}}{d t} & =-\frac{9}{2} A_{2}+3 \pi B_{2}+144 \pi^{2} h_{0} A_{4}-64 \pi^{3} h_{0} B_{4}-16 \pi^{4} h_{0} A_{3}, \\
\frac{d B_{2}}{d t} & =-3 B_{2}-48 \pi h_{0} A_{4}+48 \pi^{2} h_{0} B_{4}+32 \pi^{3} h_{0} A_{3}-16 \pi^{4} h_{0} B_{3} .
\end{aligned}
$$

Considering (3.12) and using (3.10) in the form

$$
\begin{aligned}
& A(x, t)=\frac{a_{1}(t) \beta}{b_{1}(t)+c_{1}(t) \beta+d_{1}(t) \beta^{2}+x^{2}}, \\
& B(x, t)=\frac{a_{2}(t)+b_{2}(t) \beta+x^{2}}{c_{2}(t)+d_{2}(t) \beta+e_{2}(t) \beta^{2}+x^{2}},
\end{aligned}
$$


we can express $a_{i}(t), b_{j}(t), c_{k}(t), d_{l}(t)$, and $e_{m}(t)$ in terms of the known $A_{i}(t), B_{j}(t)$ as outlined in the previous subsection, giving

$$
\begin{gathered}
a_{1}=\frac{-16 \pi^{3} h_{0}}{3}, \quad b_{1}=\frac{-8+71 h_{0}^{3}-38 h_{0}^{6}}{18 \pi^{2} h_{0}^{6}}, \quad c_{1}=\frac{-8\left(-2+19 h_{0}^{3}\right) \pi^{2}}{9 h_{0}^{2}}, \\
d_{1}=\frac{256 h_{0}^{2} \pi^{6}}{9}, \quad a_{2}=\frac{7}{2 \pi^{2}}, \quad b_{2}=\frac{-16 h_{0} \pi^{2}}{3}, \\
c_{2}=\frac{-32+5 h_{0}^{3}+118 h_{0}^{6}}{18 \pi^{2} h_{0}^{6}}, \quad d_{2}=\frac{8 \pi^{2}\left(2-11 h_{0}^{3}\right)}{3 h_{0}^{2}}, \quad e_{2}=\frac{256 h_{0}^{2} \pi^{6}}{9} .
\end{gathered}
$$

The solution of $A(x, t)$ and $B(x, t)$ is given by (3.13), respectively, where $a_{i}(t), b_{j}(t)$, $c_{k}(t), d_{l}(t)$, and $e_{m}(t)$ are defined by (3.14).

3.2.2. Solution for all $x, t$. In this subsection, we solve for $h_{1}(x, t)$ including time dependence over the entire $x$ domain. We will neglect space derivatives and justify this a posteriori. Substituting (3.1) into (2.21), we obtain

$$
\begin{aligned}
\frac{\partial A}{\partial t} x & \sin (2 \pi x)+\frac{\partial B}{\partial t} \cos (2 \pi x) \\
= & \left(-\frac{9}{2} h_{0}^{2} A-3 \pi h_{0}^{2}+3 \pi h_{0}^{2} B-16 \pi^{4} \beta h_{0}^{3} A\right) x \sin (2 \pi x) \\
& +\left(3 h_{0}^{2}-3 h_{0}^{2} B+32 \pi^{3} \beta h_{0}^{3} A-16 \pi^{4} \beta h_{0}^{3} B-3 \pi h_{0}^{2} A x^{2}\right) \cos (2 \pi x) .
\end{aligned}
$$

By equating the coefficients of $\sin (2 \pi x)$ and $\cos (2 \pi x)$ on both sides of (3.15), we obtain a pair of coupled pseudopartial differential equations

$$
\begin{aligned}
& \frac{\partial A(x, t)}{\partial t}+\alpha_{1}(t) A(x, t)+\beta_{1}(t) B(x, t)=r_{1}(t), \\
& \frac{\partial B(x, t)}{\partial t}+\alpha_{2}(x, t) A(x, t)+\beta_{2}(t) B(x, t)=r_{2}(t),
\end{aligned}
$$

where

$$
\begin{array}{lll}
\alpha_{1}=\frac{9}{2} h_{0}^{2}+16 \pi^{4} \beta h_{0}^{3}, & \beta_{1}=-3 \pi h_{0}^{2}, & r_{1}=-3 \pi h_{0}^{2}, \\
\alpha_{2}=-32 \pi^{3} \beta h_{0}^{3}+3 \pi h_{0}^{2} x^{2}, & \beta_{2}=3 h_{0}^{2}+16 \pi^{4} \beta h_{0}^{3}, & r_{2}=3 h_{0}^{2} .
\end{array}
$$

The time dependence of the coefficients (3.18) in (3.16) and (3.17) is solely incorporated in $h_{0}$ as can be seen from (2.17). Substituting (3.16) into (3.17), we obtain

$$
\begin{aligned}
\frac{\partial^{2} A}{\partial t^{2}} & +\left(\alpha_{1}-\frac{1}{\beta_{1}} \frac{\partial \beta_{1}}{\partial t}+\beta_{2}\right) \frac{\partial A}{\partial t}+\left(\frac{\partial \alpha_{1}}{\partial t}-\beta_{1} \alpha_{2}-\alpha_{1}\left(\frac{1}{\beta_{1}} \frac{\partial \beta_{1}}{\partial t}-\beta_{2}\right)\right) A \\
& =\frac{\partial r_{1}}{\partial t}-r_{1}\left(\frac{1}{\beta_{1}} \frac{\partial \beta_{1}}{\partial t}-\beta_{2}\right)-\beta_{1} r_{2} .
\end{aligned}
$$

We substitute (3.18) into the coefficients of (3.19) and use (2.17) and the chain rule to change the independent variable $t$ to $h_{0}$, giving

$$
\frac{\partial^{2} A}{\partial h_{0}^{2}}-\left(32 \pi^{4} \beta h_{0}^{3}+\frac{2}{h_{0}}\right) \frac{\partial A}{\partial h_{0}}+\left(9 \pi^{2} h_{0}^{4} x^{2}+256 \pi^{8} \beta^{2} h_{0}^{6}-16 \pi^{4} \beta h_{0}^{2}\right) A=-48 \pi^{5} \beta h_{0}^{5} .
$$


In the appendix, we show that

$$
\begin{aligned}
A(x, t)=\frac{8 \sqrt{2} \pi^{5} \beta}{\sqrt{x}} h_{0}^{3 / 2} e^{4 \pi^{4} \beta h_{0}^{4}}[ & -J_{1 / 2}\left(\pi x h_{0}^{3}\right) \int_{h_{0}}^{\infty} \bar{h}_{0}^{3} e^{-4 \pi^{4} \beta \bar{h}_{0}^{4}} \cos \left(\pi x \bar{h}_{0}^{3}\right) d \bar{h}_{0} \\
& \left.+J_{-1 / 2}\left(\pi x h_{0}^{3}\right) \int_{h_{0}}^{\infty} \bar{h}_{0}^{3} e^{-4 \pi^{4} \beta \bar{h}_{0}^{4}} \sin \left(\pi x \bar{h}_{0}^{3}\right) d \bar{h}_{0}\right] .
\end{aligned}
$$

We obtain an expression for $B(x, t)$ by substitution using (3.16) and (2.17) and find, after simplification,

$$
\begin{aligned}
B(x, t)=1 & -8 \sqrt{2} \pi^{5} \beta \sqrt{x}\left(1+h_{0}^{3}\right) h_{0}^{3 / 2} e^{4 \pi^{4} \beta h_{0}^{4}} \\
\times & {\left[-J_{1 / 2}^{\prime}\left(\pi x h_{0}^{3}\right) \int_{h_{0}}^{\infty} \bar{h}_{0}^{3} e^{-4 \pi^{4} \beta \bar{h}_{0}^{4}} \cos \left(\pi x \bar{h}_{0}^{3}\right) d \bar{h}_{0}\right.} \\
& \left.+J_{-1 / 2}^{\prime}\left(\pi x h_{0}^{3}\right) \int_{h_{0}}^{\infty} \bar{h}_{0}^{3} e^{-4 \pi^{4} \beta \bar{h}_{0}^{4}} \sin \left(\pi x \bar{h}_{0}^{3}\right) d \bar{h}_{0}\right] \\
+ & {\left[\frac{1}{3 \pi}\left(\frac{9}{2}+16 \pi^{4} \beta h_{0}\right)-\frac{1+h_{0}^{3}}{3 \pi h_{0}^{3}}\left(\frac{3}{2}+16 \pi^{4} \beta h_{0}^{4}\right)\right] A(x, t) . }
\end{aligned}
$$

In Figures 3.1, 3.2, 3.3, and 3.4, we graphed $h_{1}(x, t)$ (using the solutions of Section 3.2.2) against $x$ at $t=0.1,0.3$, respectively, with $\beta=10,20$. We found excellent agreement between $h_{1}(x, t)$ of Sections 3.2.1 and 3.2.2 when $x \ll \beta$. We also found agreement between the time-dependent solutions for large $t$ and the quasisteady solutions. Consequently, the a posteriori verification that justifies neglecting the spatial derivatives of $A(x)$ and $B(x)$ in Section 3.1.1 also suffices for Section 3.2.2.

Despite the different approaches used, all four solutions are similar though the last solution is the most complete since it includes time dependence and is valid for all $x$ (unlike the solution methods in Sections 3.1.2 and 3.2.1).

For $x \ll \beta, t \gg 1$, Section 3.1.2 is appropriate. For other values of $x$, the solution given by Section 3.1.1 should be selected. For $x \ll \beta$, the solution outlined by Section 3.2.1 is the simplest. For other $x$ values, the solution given by Section 3.2.2 should be chosen.

Finally, in Figure 3.5, by way of partial verification of the approach taken in this paper, we compare the solutions of Section 3.1.1 to a perturbation solution for $t \gg 1$, $\beta \ll 1$. The perturbation solution (chosen to be regular at the origin) is

$$
h_{1}=\cos (2 \pi x)+\frac{-8 \beta \pi^{2} h_{0}}{3 x^{2}}(\cos (2 \pi x)+2 \pi x \sin (2 \pi x)-1)+O\left(\beta^{2}\right)
$$

and it is clear that there is very good agreement between the solutions.

4. The fluid profile over an arbitrary even topography. Since we have obtained the fluid profile over a particular sinusoidal topography, we can use Fourier theory to find the fluid thickness over an arbitrary even periodic topography. Such topographies appear commonly in industry, for example, when applying the second and third phosphor colours to the inside of a TV screen [12]. Taking an arbitrary even topography of period 


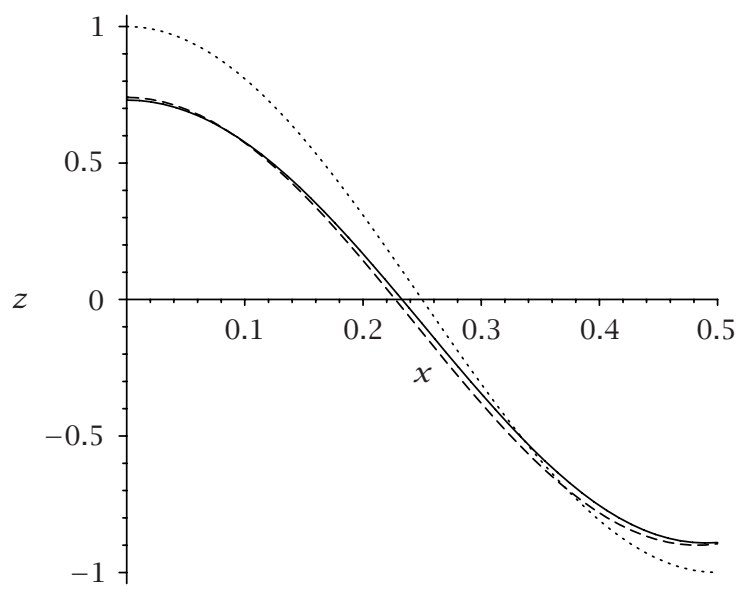

\begin{tabular}{l} 
Section 3.1 .1 \\
\hline----- Perturbation solution \\
-......... Topography
\end{tabular}

FIgURE 3.5. $h_{1}(x, t), t \gg 1$. Comparisons of $h_{1}$ using Section 3.1.1 and a perturbation approach. $h_{0}=1, \beta=0.001$.

2Q, $f\left(x^{*}\right)$, then

$$
f\left(x^{*}\right)=c_{0}+\sum_{n=1}^{\infty} c_{n} \cos \frac{n \pi}{Q} x^{*} d x^{*}
$$

where

$$
c_{0}=\frac{1}{Q} \int_{0}^{Q} f\left(x^{*}\right) d x^{*}, \quad c_{n}=\frac{2}{Q} \int_{0}^{Q} f\left(x^{*}\right) \cos \frac{n \pi}{Q} x^{*} d x^{*} .
$$

The solution for (2.21) when the topography is

$$
\cos 2 \pi x \text { or } \cos \frac{2 \pi x^{*}}{L}
$$

is given by (3.1). At this point, we rewrite (3.1) in dimensional form. If we replace $L$ by $2 Q / n$ in (3.1), we have a solution for the topography $\cos (n \pi / Q) x^{*}$ which we define as $h_{1}^{[n]}$. Since (2.21) is linear, the solution for an arbitrary topography $f\left(x^{*}\right)$ is

$$
h_{1}^{f\left(x^{*}\right)}=\sum_{n=1}^{\infty} c_{n} h_{1}^{[n]}
$$

$c_{0}$ does not appear in (4.4) as it has no effect on the first-order perturbation term of the fluid profile. 
5. Summary. In this paper, we found the fluid profile flowing over a particular small sinusoidal topography during spin-coating. We used lubrication theory to obtain an evolution equation for the fluid thickness. We then applied a perturbation technique to solve for the fluid profile and we found a translation-invariant solution for the fluid thickness which is dependent on time and perpendicular distance from the central line (the line parallel to the peaks of the topography and passing through the axis of rotation).

Using a number of ad hoc analytical techniques, we solved for the first-order perturbation term of the fluid thickness. In Section 3.1.1, we solved for the fluid profile over the entire substrate for $t \gg 1$. In Section 3.1.2, we found the steady state solution in the region close to the central line. Neglecting time dependence is justifiable close to the end of the process as a quasisteady state is approached. In Section 3.2.1, we include time dependence but restrict the solution domain to that of Section 3.1.2. In Section 3.2.2, we find a time-dependent solution over the entire substrate.

Despite the different solution methods, the two time-independent solutions and the two time-dependent solutions of $A(x, t)$ and $B(x, t)$ and thus $h_{1}(x, t)$ are almost identical as can be seen in Figures 3.1, 3.2, 3.3, and 3.4. The last solution method is the most complete as it includes time dependence (unlike solution methods in Sections 3.1.1 and 3.1.2) and is valid over the entire substrate. Neglecting the space derivatives of $A(x, t)$ and $B(x, t)$ was justified a posteriori.

Any substrate topography which is periodic in one Cartesian coordinate and independent of the other can be represented by a Fourier series. Since the equation for the first-order perturbation term of the scaled fluid thickness (2.21) is linear, the fluid profile over such a topography can be obtained as a sum of solutions of fluid thicknesses over sinusoidal topographies with different periods. Consequently, this analysis gives fluid profile solutions over real topographies which occur in industry, for example, the topography which occurs when applying the second and third phosphor colours during spin-coating of TV screens [10, 11, 12].

Further work should involve a detailed comparison with experimental results and should numerically verify the solutions obtained in this paper. We do not undertake the numerics here but we note that the different approximations used are, at any rate, self-consistent and physically reasonable when compared with (limited) existing experimental data $[10,11]$.

\section{Appendix}

Solutions for $A(x, t)$ and $B(x, t)$.

$$
\begin{aligned}
\frac{\partial^{2} A}{\partial h_{0}^{2}} & -\left(32 \pi^{4} \beta h_{0}^{3}+\frac{2}{h_{0}}\right) \frac{\partial A}{\partial h_{0}} \\
& +\left(9 \pi^{2} h_{0}^{4} x^{2}+256 \pi^{8} \beta^{2} h_{0}^{6}-16 \pi^{4} \beta h_{0}^{2}\right) A=-48 \pi^{5} \beta h_{0}^{5} .
\end{aligned}
$$

We will compare the homogeneous form of (A.1) to 


$$
\begin{aligned}
x^{2} \frac{d^{2} y}{d x^{2}} & -x\left(2 a+\gamma-1+2 b c x^{c}\right) \frac{d y}{d x} \\
& +\left(a(a+\gamma)+\left(\frac{1}{4}-m^{2}\right) \gamma^{2}+b c(2 a+\gamma-c) x^{c}+b^{2} c^{2} x^{2 c}+-\frac{1}{4} \alpha^{2} \gamma^{2} x^{2 \gamma}\right) y=0 .
\end{aligned}
$$

The solution of (A.2) is given by [9] as follows:

$$
y(x)=x^{a} \exp \left(b x^{c}\right) \bar{y}\left(m, \alpha x^{\gamma}\right),
$$

with

$$
\bar{y}(m, x)=C_{1} M_{m}(x)+C_{2} M_{-m}(x),
$$

where $C_{1}$ and $C_{2}$ are constants and

$$
M_{m}(x)=\sqrt{x} J_{m}\left(\frac{i x}{2}\right) .
$$

Comparing the coefficients of the homogeneous form of (A.1) with those of (A.2), we have the following relationships:

$$
\begin{gathered}
2 a+\gamma-1=2, \quad c=4, \quad 2 b c=32 \pi^{4} \beta, \\
a(a+\gamma)+\left(\frac{1}{4}-m^{2}\right) \gamma^{2}=0, \quad b c(2 a+\gamma-c)=-16 \pi^{4} \beta, \\
b^{2} c^{2}=256 \pi^{8} \beta^{2}, \quad \gamma=3, \quad-\frac{1}{4} \alpha^{2} \gamma^{2}=9 \pi^{2} x^{2} .
\end{gathered}
$$

As 6 of the 8 equations in (A.6) are independent, we can solve for the 6 unknowns $a$, $b, c, \alpha, \gamma$, and $m$, giving

$$
a=0, \quad b=4 \pi^{4} \beta, \quad c=4, \quad \alpha= \pm 2 \pi x i, \quad \gamma=3, \quad m= \pm \frac{1}{2} .
$$

Using (A.3), (A.4), (A.5), and (A.7), the two fundamental solutions of the homogeneous form of (A.1) which we define as $u_{1}$ and $u_{2}$ are

$$
\begin{aligned}
& u_{1}=\sqrt{x} \exp \left(4 \pi^{4} \beta h_{0}^{4}\right) h_{0}^{3 / 2} J_{1 / 2}\left(\pi x h_{0}^{3}\right), \\
& u_{2}=\sqrt{x} \exp \left(4 \pi^{4} \beta h_{0}^{4}\right) h_{0}^{3 / 2} J_{-1 / 2}\left(\pi x h_{0}^{3}\right) .
\end{aligned}
$$

Using "variation of parameters," we will find a solution to the inhomogeneous form of (A.1) of the form

$$
A=u_{1} v_{1}+u_{2} v_{2}
$$

where

$$
u_{1} v_{1}^{\prime}+u_{2} v_{2}^{\prime}=0, \quad u_{1}^{\prime} v_{1}^{\prime}+u_{2}^{\prime} v_{2}^{\prime}=-48 \pi^{5} \beta h_{0}^{5} .
$$

Solving (A.10) for $v_{1}^{\prime}$ and $v_{2}^{\prime}$, we obtain

$$
v_{1}^{\prime}=\frac{48 \pi^{5} \beta h_{0}^{5} u_{2}}{u_{1} u_{2}^{\prime}-u_{1}^{\prime} u_{2}}, \quad v_{2}^{\prime}=\frac{-48 \pi^{5} \beta h_{0}^{5} u_{1}}{u_{1} u_{2}^{\prime}-u_{1}^{\prime} u_{2}}
$$


The denominator of (A.11) is

$$
3 \pi x^{2} h_{0}^{5} \exp \left(8 \pi^{4} \beta h_{0}^{4}\right)\left(J_{1 / 2}\left(\pi x h_{0}^{3}\right) J_{-1 / 2}^{\prime}\left(\pi x h_{0}^{3}\right)-J_{1 / 2}^{\prime}\left(\pi x h_{0}^{3}\right) J_{-1 / 2}\left(\pi x h_{0}^{3}\right)\right),
$$

which can be written as

$$
3 \pi x^{2} h_{0}^{5} \exp \left(8 \pi^{4} \beta h_{0}^{4}\right) W\left[J_{1 / 2}\left(\pi x h_{0}^{3}\right), J_{-1 / 2}\left(\pi x h_{0}^{3}\right)\right]
$$

where $W$ is the Wronskian of the two functions. Since

$$
W\left[J_{1 / 2}(z), J_{-1 / 2}(z)\right]=\frac{-2}{\pi z},
$$

(A.12) can be written as

$$
-\frac{6}{\pi} x h_{0}^{2} \exp \left(8 \pi^{4} \beta h_{0}^{4}\right)
$$

Substituting (A.15) into (A.11), we have

$$
\begin{aligned}
& v_{1}^{\prime}=-8 \pi^{6} \beta \frac{h_{0}^{9 / 2}}{\sqrt{x}} \exp \left(-4 \pi^{4} \beta h_{0}^{4}\right) J_{-1 / 2}\left(\pi x h_{0}^{3}\right), \\
& v_{2}^{\prime}=8 \pi^{6} \beta \frac{h_{0}^{9 / 2}}{\sqrt{x}} \exp \left(-4 \pi^{4} \beta h_{0}^{4}\right) J_{1 / 2}\left(\pi x h_{0}^{3}\right) .
\end{aligned}
$$

Since

$$
J_{-1 / 2}(x)=\sqrt{\frac{2}{\pi x}} \cos x, \quad J_{1 / 2}(x)=\sqrt{\frac{2}{\pi x}} \sin x,
$$

(A.16) can be written as

$$
\begin{aligned}
& v_{1}^{\prime}=-8 \sqrt{2} \pi^{5} \beta \frac{h_{0}^{3}}{x} \exp \left(-4 \pi^{4} \beta h_{0}^{4}\right) \cos \left(\pi x h_{0}^{3}\right), \\
& v_{2}^{\prime}=8 \sqrt{2} \pi^{5} \beta \frac{h_{0}^{3}}{x} \exp \left(-4 \pi^{4} \beta h_{0}^{4}\right) \sin \left(\pi x h_{0}^{3}\right) .
\end{aligned}
$$

Using (A.9), (A.8), and (A.18), we obtain

$$
\begin{aligned}
A(x, t)=\frac{8 \sqrt{2} \pi^{5} \beta}{\sqrt{x}} h_{0}^{3 / 2} e^{4 \pi^{4} \beta h_{0}^{4}}[ & -J_{1 / 2}\left(\pi x h_{0}^{3}\right) \int_{h_{0}}^{\infty} \bar{h}_{0}^{3} e^{-4 \pi^{4} \beta \bar{h}_{0}^{4}} \cos \left(\pi x \bar{h}_{0}^{3}\right) d \bar{h}_{0} \\
& \left.+J_{-1 / 2}\left(\pi x h_{0}^{3}\right) \int_{h_{0}}^{\infty} \bar{h}_{0}^{3} e^{-4 \pi^{4} \beta \bar{h}_{0}^{4}} \sin \left(\pi x \bar{h}_{0}^{3}\right) d \bar{h}_{0}\right] .
\end{aligned}
$$


We obtain an expression for $B(x, t)$ by substitution using (3.16) and (2.17) and find, after simplification,

$$
\begin{aligned}
B(x, t)=1 & -8 \sqrt{2} \pi^{5} \beta \sqrt{x}\left(1+h_{0}^{3}\right) h_{0}^{3 / 2} e^{4 \pi^{4} \beta h_{0}^{4}} \\
\times & {\left[-J_{1 / 2}^{\prime}\left(\pi x h_{0}^{3}\right) \int_{h_{0}}^{\infty} \bar{h}_{0}^{3} e^{-4 \pi^{4} \beta \bar{h}_{0}^{4}} \cos \left(\pi x \bar{h}_{0}^{3}\right) d \bar{h}_{0}\right.} \\
& \left.+J_{-1 / 2}^{\prime}\left(\pi x h_{0}^{3}\right) \int_{h_{0}}^{\infty} \bar{h}_{0}^{3} e^{-4 \pi^{4} \beta \bar{h}_{0}^{4}} \sin \left(\pi x \bar{h}_{0}^{3}\right) d \bar{h}_{0}\right] \\
+ & {\left[\frac{1}{3 \pi}\left(\frac{9}{2}+16 \pi^{4} \beta h_{0}\right)-\frac{1+h_{0}^{3}}{3 \pi h_{0}^{3}}\left(\frac{3}{2}+16 \pi^{4} \beta h_{0}^{4}\right)\right] A(x, t) . }
\end{aligned}
$$

\section{REFERENCES}

[1] D. J. Acheson (ed.), Elementary Fluid Dynamics, Oxford Applied Mathematics and Computing Science Series, Clarendon Press, Oxford University Press, New York, 1990.

[2] R. H. de Bruijne and J. Lammers, Experiments on finger formation during spin coating, Proc. 3rd Eur. Coating Symp., Erlangen-Nürnberg, Erlangen, 1999.

[3] A. G. Emslie, F. T. Bonner, and L. G. Peck, Flow of a viscous liquid on a rotating disk, J. Appl. Phys. 29 (1958), no. 5, 858-862.

[4] C. M. Gramlich, S. Kalliadasis, G. M. Homsy, and C. Messer, Optimal leveling of flow over one-dimensional topography by Marangoni stresses, Phys. Fluids 14 (2002), no. 6, 1841-1850.

[5] M. Hayes, S. B. G. O’Brien, and J. H. Lammers, Green's function for steady flow over a small two-dimensional topography, Phys. Fluids 12 (2000), no. 11, 2845-2858.

[6] J. H. Hwang and F. Ma, On the flow of a thin liquid over a rough rotating disk, J. Appl. Phys. 66 (1989), no. 1, 388-394.

[7] S. Kalliadasis, C. Bielarz, and G. M. Homsy, Steady free-surface thin film flows over topography, Phys. Fluids 12 (2000), no. 8, 1889-1898.

[8] S. Kalliadasis and G. M. Homsy, Stability of free-surface thin-film flows over topography, J. Fluid Mech. 448 (2001), 387-410.

[9] E. Kamke, Differentialgleichungen, Mathematik und ihre Anwendungen in Physik und Technik. Reihe A., vol. 18, Akademische Verlagsgesellschaft Geest \& Portig K.-G., Leipzig, 1959.

[10] J. H. Lammers, Effects of evaporation during the spin coating process, Nat. Lab. Technical Note Nr.182/94, Philips Research Labs., The Netherlands, 1994.

[11] _ Analysis of spin-coating over topography, Nat. Lab. Technical Note Nr.337/95, Philips Research Labs., The Netherlands, 1995.

[12] J. H. Lammers, S. B. G. O’Brien, and M. M. J. Decré, Spin-coating over topography, Proc. 2nd Eur. Coating Symp. (Strasbourg), 1997, pp. 278-290.

[13] R. G. Larson and T. J. Rehg, Spin coating, Liquid Film Coating (S. F. Kistler and P. M. Schweizer, eds.), Chapman \& Hall, London, 1997, pp. 709-734.

[14] C. J. Lawrence, The mechanics of spin coating of polymer films, Phys. Fluids 31 (1988), no. 10, 2786-2795.

[15] _ Spin coating with slow evaporation, Phys. Fluids A 2 (1990), no. 3, 453-456.

[16] C. C. Lim and L. Sirovich, Nonlinear vortex trail dynamics, Phys. Fluids 31 (1988), no. 5, 991-998.

[17] - Nonlinear vortex trail dynamics. II. Analytic solutions, Quart. Appl. Math. 51 (1993), no. 1, 129-146.

[18] L. M. Manske and D. B. Graves, Origins of asymmetry in spin-cast films over topography, Optical/Laser Microlithography IV, Proceedings of SPIE, vol. 1463, SPIE, Washington, 1991, pp. 414-422. 
[19] D. Meyerhofer, Characteristics of resist films produced by spinning, J. Appl. Phys. 49 (1978), no. 7, 3993-3997.

[20] W. M. Moreau, Semiconductor Lithography, Plenum Press, New York, 1988.

[21] T. G. Myers, Thin films with high surface tension, SIAM Rev. 40 (1998), no. 3, 441-462.

[22] S. B. G. O'Brien and M. Hayes, A model for dip-coating of a two liquid mixture, Int. J. Math. Math. Sci. 29 (2002), no. 6, 313-324.

[23] L. M. Peurrung and D. B. Graves, Film thickness profiles over topography in spin coating, J. Electrochem. Soc. 138 (1991), no. 7, 2115-2124.

[24] C. Pozrikidis, The flow of a liquid film along a periodic wall, J. Electrochem. Soc. 188 (1988), 275-300.

[25] C. Pozrikidis and S. T. Thoroddsen, The deformation of a liquid film flowing down an inclined plane wall over a small particle arrested on the wall, Phys. Fluids A 3 (1991), no. 11, 2546-2558.

[26] L. W. Schwartz and D. E. Weidner, Modeling of coating flows on curved surfaces, J. Engrg. Math. 29 (1995), no. 1, 91-103.

[27] L. E. Stillwagon and R. G. Larson, Levelling of thin films over uneven substrate during spincoating, Phys. Fluids A 2 (1990), no. 11, 1937-1944.

[28] P. C. Sukanek, A model for spin coating with topography, J. Electrochem. Soc. 136 (1989), no. 10, 3019-3026.

[29] V. Suresh and G. M. Homsy, Erratum: Stability of return thermocapillary flows under gravity modulation, Phys. Fluids 14 (2002), no. 2, 910.

M. A. Hayes: Department of Mathematics, National University of Ireland, Galway, Ireland E-mail address: mi chae1. hayes@nuiga7way. ie

S. B. G. O’Brien: Department of Mathematics and Statistics, University of Limerick, Limerick, Ireland

E-mail address: stephen.obrien@u1.ie 


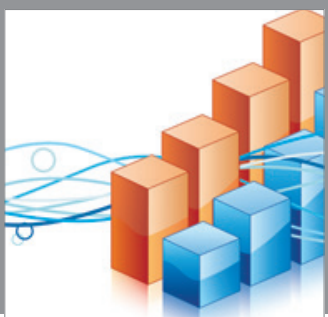

Advances in

Operations Research

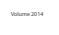

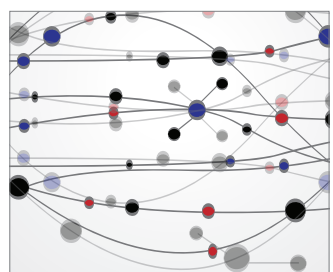

\section{The Scientific} World Journal
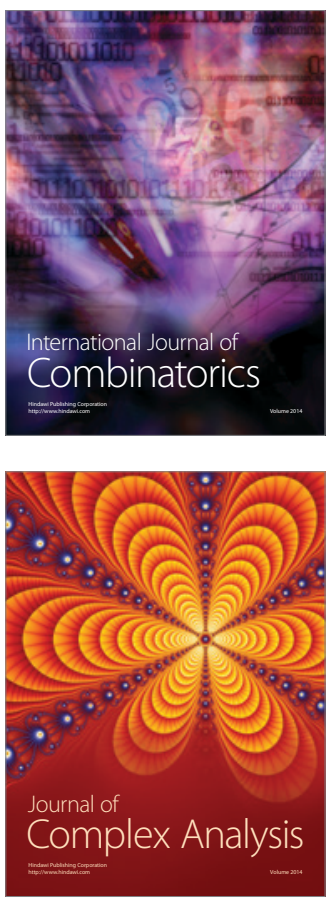

International Journal of

Mathematics and

Mathematical

Sciences
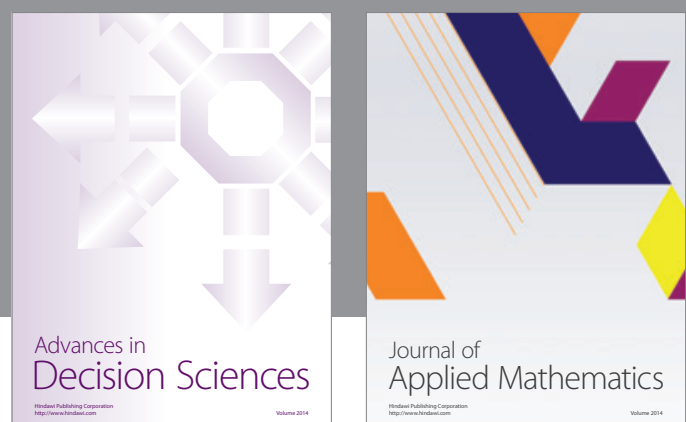

Journal of

Applied Mathematics
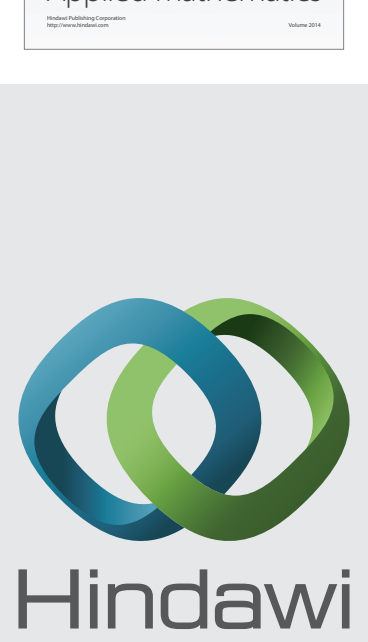

Submit your manuscripts at http://www.hindawi.com
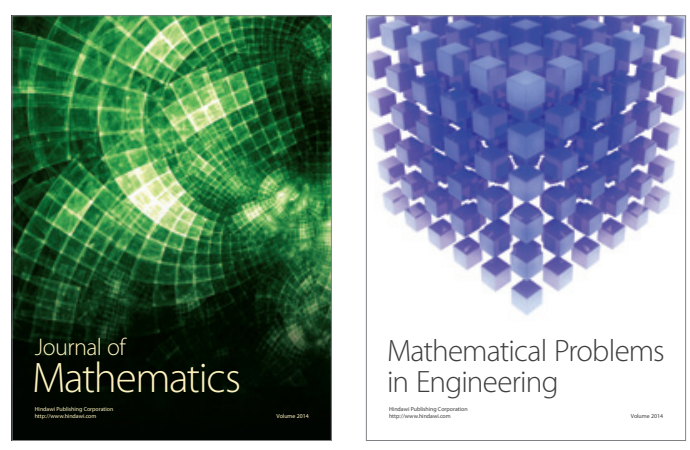

Mathematical Problems in Engineering
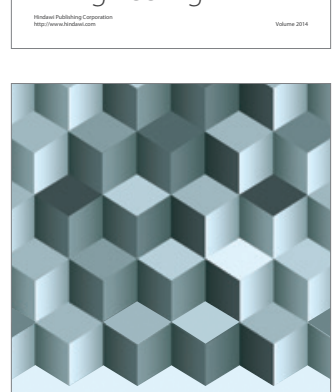

Journal of

Function Spaces
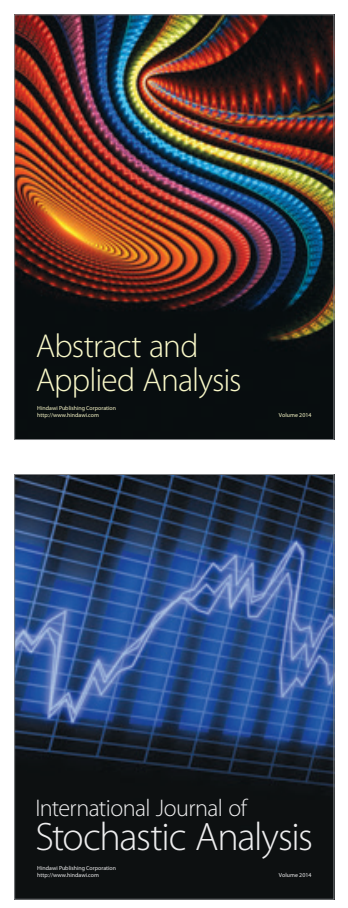

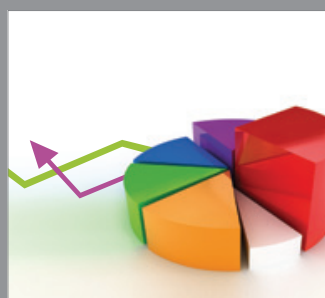

ournal of

Probability and Statistics

Promensencen
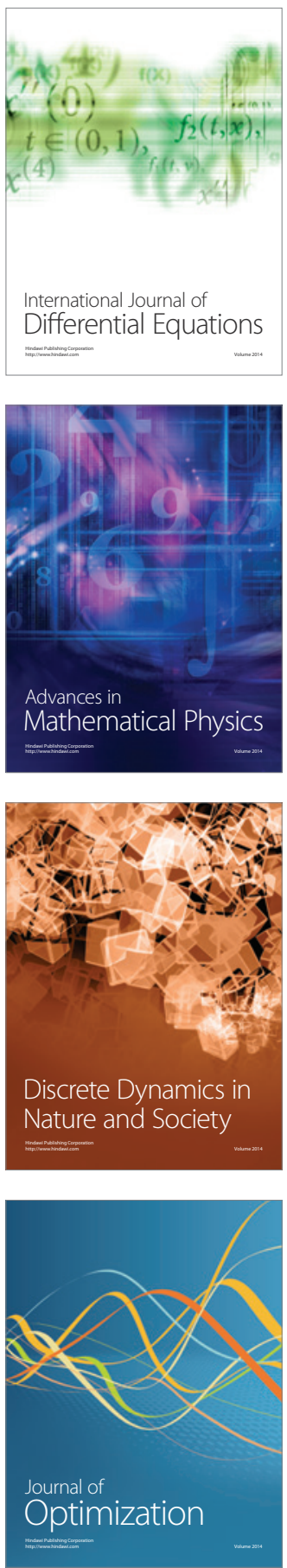